\title{
Locus Ceruleus Regulates Sensory Encoding by Neurons and Networks in Waking Animals
}

\author{
David M. Devilbiss, ${ }^{1}$ Michelle E. Page, ${ }^{2}$ and Barry D. Waterhouse ${ }^{3}$ \\ ${ }^{1}$ Department of Psychology, University of Wisconsin-Madison, Madison, Wisconsin 53706, ${ }^{2}$ Department of Neurosurgery, Thomas Jefferson University, \\ Philadelphia, Pennsylvania 19107, and ${ }^{3}$ Department of Neurobiology and Anatomy, Drexel University, Philadelphia, Pennsylvania 19129
}

\begin{abstract}
Substantial evidence indicates that the locus ceruleus (LC)-norepinephrine (NE) projection system regulates behavioral state and statedependent processing of sensory information. Tonic LC discharge (0.1-5.0 Hz) is correlated with levels of arousal and demonstrates an optimal firing rate during good performance in a sustained attention task. In addition, studies have shown that locally applied NE or LC stimulation can modulate the responsiveness of neurons, including those in the thalamus, to nonmonoaminergic synaptic inputs. Many recent investigations further indicate that within sensory relay circuits of the thalamus both general and specific features of sensory information are represented within the collective firing patterns of like-modality neurons. However, no studies have examined the impact of NE or LC output on the discharge properties of ensembles of functionally related cells in intact, conscious animals. Here, we provide evidence linking LC neuronal discharge and NE efflux with LC-mediated modulation of single-neuron and neuronal ensemble representations of sensory stimuli in the ventral posteriomedial thalamus of waking rats. As such, the current study provides evidence that output from the LC across a physiologic range modulates single thalamic neuron responsiveness to synaptic input and representation of sensory information across ensembles of thalamic neurons in a manner that is consistent with the well documented actions of LC output on cognition.
\end{abstract}

Key words: multichannel single unit recording; neuronal ensemble activity; sensory information processing; neuron; somatosensory thalamus; principal components analysis

\section{Introduction}

The ability to regulate information processing under diverse behavioral conditions and ever changing motivational contingencies is an essential property of the CNS. Considerable evidence indicates that the locus ceruleus (LC)-norepinephrine (NE) projection system regulates behavioral state and state-dependent processing of sensory information (Foote et al., 1983; Berridge and Waterhouse, 2003). The LC exhibits tonic discharge, characterized by a state-dependent, relatively slow $(0.1-5.0 \mathrm{~Hz})$ and highly regular pattern of firing. Increasing tonic LC output activates the forebrain, as measured by EEG (Berridge and Foote, 1991), and is correlated with arousal levels (Foote et al., 1980; Aston-Jones and Bloom, 1981), and progression from drowsy, low-attention states to alert, highly vigilant states (Rajkowski et al., 1994; Usher et al., 1999).

The moderately dense projection of NE-containing LC efferent fibers (Simpson et al., 1997) is capable of simultaneously releasing NE in subcortical and cortical circuits that sequentially process like-modality sensory information (e.g., the rodent tri-

Received March 9, 2004; revised Aug. 11, 2006; accepted Aug. 14, 2006

This work was supported by National Institutes of Health Grant NS 32461 (B.D.W.). We thank Drs. Craig Berridge John Chapin, and Rita Valentino for timely discussions and helpful comments throughout various stages of this work and Ron Markowitz for help with electrophysiological data collection.

Correspondence should be addressed to David M. Devilbiss, Department of Psychology, 1202 West Johnson Street, Room 523, Madison, Wisconsin 53706. E-mail: ddevilbiss@wisc.edu.

D0I:10.1523/JNEUROSCI.1776-06.2006

Copyright $\odot 2006$ Society for Neuroscience $\quad$ 0270-6474/06/269860-13\$15.00/0 geminal somatosensory pathway). Within the thalamus, exogenous or synaptically released NE produces a spectrum of cellular modulatory actions (for review, see Berridge and Waterhouse, 2003). Importantly, these diverse actions of NE occur simultaneously across thalamic neurons (Devilbiss and Waterhouse, 2002). Thus, LC activation and the resulting synaptic release of NE may place target regions of the brain, such as the thalamus, in a state of preparedness to accurately encode and respond to salient stimulus inputs.

The rat vibrissae somatosensory system has been used extensively as a model for better understanding the relationship between single-cell discharge patterns (Waite, 1973; Simons, 1978; Ito, 1988; Armstrong-James et al., 1992; Ahissar et al., 2000; Shoykhet et al., 2000), ensemble neuronal activity (Nicolelis et al., 1995; Ahissar et al., 1997; Petersen and Diamond, 2000; Petersen et al., 2001, 2002; Panzeri et al., 2003), and central coding of sensory information from peripheral receptors (Chapin and Nicolelis, 1999; Panzeri et al., 2001). Within "barreloid" specializations of the ventral posteriomedial (VPM) thalamus (Van der Loos, 1976), individual neurons respond to deflection of a single preferred whisker on the contralateral face with discharge patterns that are coded for direction, magnitude, and velocity of movement (Waite, 1973; Shipley, 1974; Ito, 1981). Within the nervous system complex, computations and information transfer are accomplished via arrays of functionally related neurons (Erickson, 1968; Katchalsky et al., 1974; Churchland, 1989). For example, distributed activity across ensembles of thalamic cells 
represent direction of stimulus movement across multiple whiskers of the vibrissae pad (Nicolelis and Chapin, 1994; Nicolelis, 1996; Chapin and Nicolelis, 1999).

Although substantial information exists regarding the effects of the LC-NE system on sensory signal processing capabilities of individual neurons (Rogawski and Aghajanian, 1980; Waterhouse et al., 1990; Manunta and Edeline, 1997; Devilbiss and Waterhouse, 2000; Berridge and Waterhouse, 2003; Hurley et al., 2004), no studies have considered the impact of the noradrenergic system on ensemble neuronal activity in intact, conscious animals. To address this gap in our understanding, we present data indicating a link between output of the LC-NE system and modulation of the sensory response properties of individual neurons and neuronal ensembles within the VPM thalamus of waking rats. As such, these studies examined sensory information mapping across ensembles of simultaneously recorded neurons with correlation analysis and principal component analysis (PCA) of discharge patterns to infer how NE modulates sensory signals relayed to the cortex. Additionally, these studies examined microdialysis measures of extracellular NE levels within the somatosensory VPM thalamus across a range of "low-intensity, low-frequency" LC stimulus conditions that modulated sensoryevoked discharge of individual thalamic neurons. By so doing, we sought to bridge the gap between the well known state-dependent changes in tonic LC output, the established spectrum of heterogeneous cellular/membrane actions of NE, and the cognitive impact of tonic LC activation.

\section{Materials and Methods}

\section{Subjects and surgery}

Adult male Long-Evans hooded rats (Charles River Laboratories, Wilmington, MA) weighing 250-450 g were used in this study (electrophysiology, $n=6$; microdialysis, $n=7$ ) in accordance with National Institutes of Health guidelines on research animal care and the Institutional Animal Care and Use Committee of Drexel University.

Electrophysiology. Extracellular microwire electrode bundles (NB Labs, Denison, TX) were implanted unilaterally in the ventral posterior medial (VPM) thalamus, and the ipsilateral LC. Detailed methodology regarding the surgical preparation, recording strategies, and data analysis have been described previously (Devilbiss and Waterhouse, 2002). Briefly, a single electrode bundle $(n=8,50 \mu \mathrm{m}$ stainless steel Teflon coated microwires) was stereotaxically placed within the LC $(\sim 1.2$ lateral, $\sim 3.6$ caudal to the intersection of the midline and lambda; incisor bar at 11.5) and confirmed electrophysiologically by monitoring neuronal electrical activity as the recording probe was lowered $(\sim 50 \mu \mathrm{m} / \mathrm{min} ; \sim 6.0$ dorsoventral). The characteristic spontaneous discharge rate $(\sim 0.1-5 \mathrm{~Hz})$ and the biphasic response to tail pinch (Akaike, 1982) were two criteria used to identify putative LC neurons within the nucleus. A second electrode bundle was cut on a diagonal to correspond to the anatomical structure of the VPM barreloids, bound with a silk suture, and implanted into the ipsilateral VPM thalamus (flat skull; -3.3 anteroposterior, -2.8 mediolateral, and $\sim 5.5$ dorsoventral from bregma). Again, neuronal activity was monitored during insertion to evaluate the position of individual microwires as they approached the C3-whisker representation of the VPM thalamus. Lastly, a flexible bipolar stimulating electrode was implanted around a single (C3) vibrissae of the rat's whiskerpad. Electrode connectors were attached to the skull with screws and dental acrylic. The skin was loosely sealed around the dental cement and the animal was allowed to recover for 5-10 d.

Microdialysis. Animals were implanted with a microwire bundle in the LC nucleus 1 week before implantation of a microdialysis probe in the ipsilateral VPM thalamus. Concentric microdialysis probes were constructed as described previously (Abercrombie and Finlay, 1991). In vitro recovery for dialysis probes was determined before implant. Microdialysis probes were targeted to the same region in the VPM thalamus as the recording electrode bundles and were secured to the skull with skull screws and dental acrylic. The dialysis probe was connected to a fluid swivel (Instech Laboratories, Plymouth Meeting, PA) and the rat was placed in the same chamber used for electrophysiological recording. Artificial CSF [aCSF; containing the following (in mM): $147 \mathrm{NaCl}, 1.7$ $\left.\mathrm{CaCl}_{2}, 0.9 \mathrm{MgCl}_{2}, 4 \mathrm{KCl}\right]$ was continuously perfused through the probe at a rate of $1.5 \mu \mathrm{l} / \mathrm{min}$. On the following experimental day, $20 \mathrm{~min}$ dialysate samples were collected before and during LC stimulation. The amount of NE in dialysates $(15 \mu \mathrm{l})$ was determined with HPLC with electrochemical detection (HPLC-EC) (Page and Abercrombie, 1997).

\section{Experimental procedure}

Awake, freely moving animals were allowed to acclimate to the testing chamber for $2 \mathrm{~h}$ before experimental procedures began. During this time, putative single units of the VPM thalamus were discriminated using template-matching algorithms. After each experimental session, preestablished offline criteria were used to demonstrate that waveforms assigned to each discriminated "unit" originated from a single neuron. These criteria are comprised of assessments based on unit waveform properties and spike train discharge patterns (Devilbiss and Waterhouse, 2002), including (1) peak waveform voltage, (2) slope(s) of waveform from peak to peak, (3) clustering of scattergram points from the first two principal components of the waveform, and (4) spike train autocorrelgram (2 ms refractory period).

Electrophysiological experiments were initiated after online discrimination of VPM thalamic units and confirmation that units were responsive to whiskerpad stimulation. The bipolar stimulating electrode implanted around the vibrissae of the rat's whiskerpad was used to deliver biphasic pulses (range 1-3 mA; $1 \mathrm{~ms}$ duration) that were threshold for producing a rostral twitch of the same single vibrissae and for eliciting a robust excitatory discharge in a majority of the simultaneously recorded VPM thalamic units. The control condition was always presented first; 90 whiskerpad stimuli that randomly varied in time $(2 \mathrm{~s}$ mean intertrial interval, range 1.5-2.5 s) were delivered to animals to prevent stimulus habituation of the somatosensory system. After the initial baseline period, three pseudorandomized tonic LC stimulation conditions (animals received each condition in a random order) were presented, followed by a recovery condition (whisker stimulation only). LC stimulation consisting of monopolar single electrical pulses at a frequency of $0.5,1.0$, and 5.0 $\mathrm{Hz}(0.2 \mathrm{~ms}$ duration) was delivered to the LC across two of the eight wires of the microwire bundle implant. A custom head stage permitted electrical activation of the LC concurrent with LC neural activity recordings. LC stimulation current was adjusted across a range of 3 to $300 \mu \mathrm{A}$ to produce the maximal effect on target VPM thalamic neurons. During each period of LC activation, whiskerpad stimuli were presented in the same manner as control conditions; however, stimulus pulses to the LC and whiskerpad were never allowed to occur simultaneously.

Data analysis. LC neuronal firing rates and VPM neuron discharge patterns were determined from computer-stored time stamps of discriminated waveforms. Initially, rate histograms with $1 \mathrm{~ms}$ time bins were used to evaluate baseline tonic discharge of LC neurons. Sensoryevoked discharge patterns of individual VPM neurons were analyzed by quantifying peristimulus time histograms (PSTHs) as described previously in Devilbiss and Waterhouse (2002). In brief, measures of stimulus-evoked firing were calculated from PSTHs collected during periods of quiet resting for either control or LC stimulation conditions. From each PSTH, the mean probability of discharge during the whisker stimulus-evoked response was calculated; the response window was defined by Gaussian $99 \%$ confidence intervals that determined the response window beginning and end. Lastly, spike trains from all verified single neurons were examined for "unreasonable correlation between spike trains" to determine that any single neuron was not recorded from more than one electrode. If a pair of neurons demonstrated a correlation $>0.6$ across the duration of the experiment, one of the neurons was excluded from analysis.

Functional changes in the ensemble of simultaneously recorded VPM neurons during LC activation were determined by analyzing differences in neuronal correlation with all-pairwise cross-correlational analyses as well as PCA. Initially, functional relationships between neuronal pairs were determined generating cross-correlegrams from individual neural 
A.

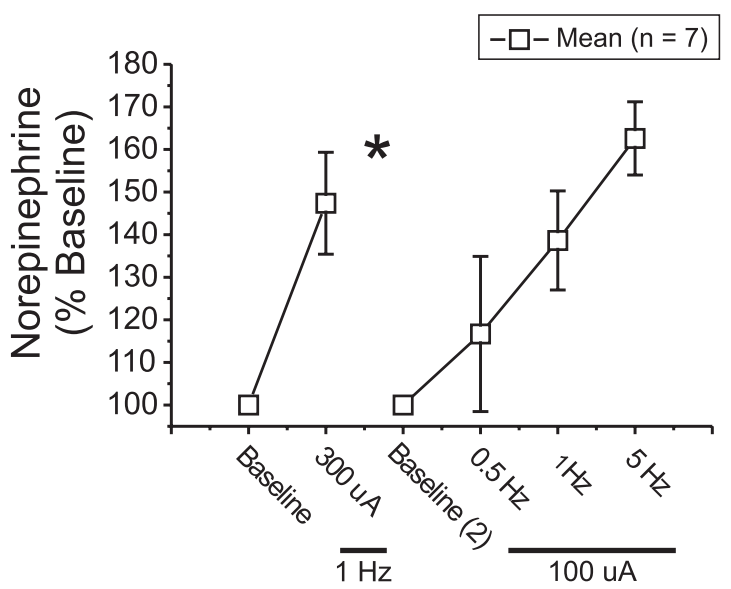

B.

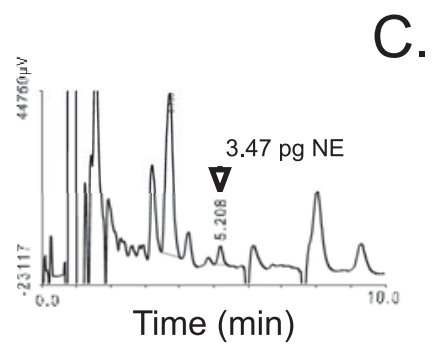

D.
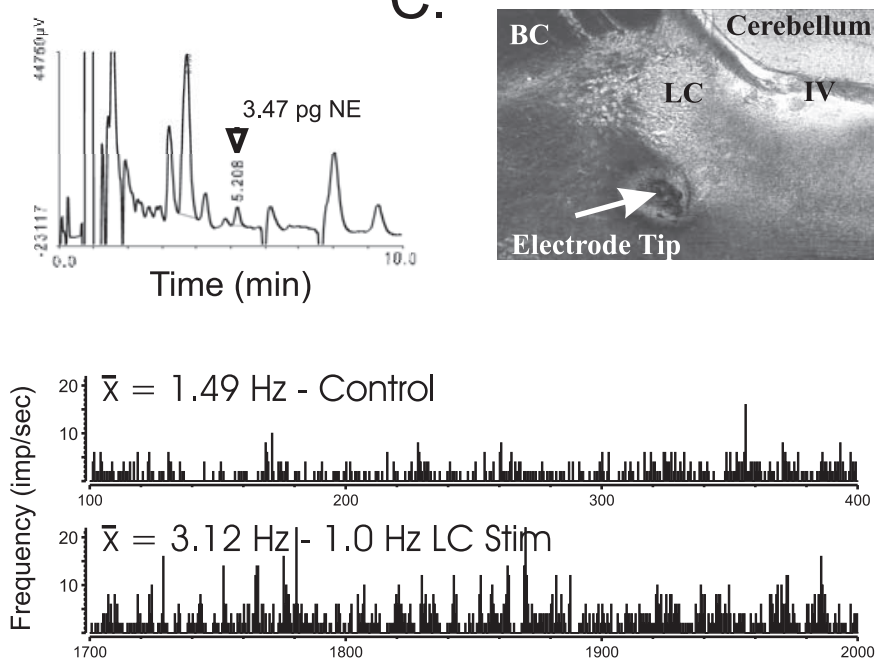

Figure 1. Relationship of tonic $\mathrm{LC}$ electrical stimulation to dialysate levels of NE and $\mathrm{LC}$ discharge rates. $A$, Graphic representation of NE levels in the thalamus with respect to ipsilateral $\mathrm{LC}$ stimulation parameters. The $y$-axis represents the concentration of NE, as determined by HPLC-EC measures, in $20 \mathrm{~min} / 15 \mu \mathrm{l}$ samples $\left({ }^{*} p<0.05\right)$. A significant increase in NE was observed (1.5-2.3 $\mathrm{pg} / 15 \mu \mathrm{l}$ ) between baseline and the $300 \mu \mathrm{A}$ at $1.0 \mathrm{~Hz}$ stimulus condition. The remaining data points were not tested for statistical significance because of possible interference from preceding data collection. Error bars indicate SE. B, Example chromatogram of dialysate sample during the $300 \mu \mathrm{A}$ at $1.0 \mathrm{~Hz} \mathrm{LC}$ stimulation condition. The $y$-axis represents microvolts of detector current, the $x$-axis represents time after sample injection, and the arrow indicates the NE signal peak. C, Photomicrograph of a brainstem coronal section. A Prussian blue reaction product indicates the placement of a recording/stimulation microwire bundle adjacent to the $L$ C nucleus. Of the cases tested, this electrode implant was the most distal from the $L C$ border but was near enough to the nucleus to produce increased levels of extracellular NE in the ipsilateral thalamus. Dorsal is up; lateral is left. $\boldsymbol{D}$, Rate histograms of $L C$ neuronal activity before and during tonic electrical stimulation of the $L C$ nucleus. For each histogram, the $y$-axis indicates discharge frequency; the $x$-axis represents experimental time (sec.). The top histogram represents a recorded unit within the $L C$ during quietly resting control conditions (no $\mathrm{LC}$ stimulation). The bottom histogram is the same unit during quiet resting behavior and $1.0 \mathrm{~Hz}$ stimulation of the $\mathrm{LC}$. The average discharge rate of the unit increased from $1.49(\boldsymbol{A})$ to $3.12 \mathrm{~Hz}(\boldsymbol{B})$.

spike train data, $3 \mathrm{~ms}$ before to $90 \mathrm{~ms}$ after whisker stimulus presentation (Gerstein et al., 1989; Devilbiss and Waterhouse, 2002). Spike trains were discretized with $3 \mathrm{~ms}$ time bins and used to calculate correlations between neural discharge patterns. Furthermore, a PSTH shift predictor was used to eliminate stimulus-induced correlation (Aertsen and Gerstein, 1985; Aertsen et al., 1989; Eggermont, 1992, 1994). Lastly, the mean correlation $10 \mathrm{~ms}$ before to $10 \mathrm{~ms}$ after each action potential was calculated and plotted as a scattergram in Matlab (MathWorks, Natick, MA). Thus, functional connectivity could be assessed from neural discharge patterns during processing of whiskerpad stimuli, but were not directly related to simultaneous activation of VPM neurons by excitatory afferent input.

Additionally, correlation matrices were used to produce an eigenvalue decomposition by PCA. Neuronal spike trains (mean frequency of $3 \mathrm{~ms}$ bin) from the entire experimental session served as variables to calculate principal components (PCs). Spike-train integration time intervals were based on the period that postsynaptic neurons (i.e., cortical neurons) likely integrate afferent input (Wilent and Contreras, 2004). Similar results were obtained at longer windows (10 ms; data not shown). An eigenfunction for each PC was calculated as the sum of eigenvalueweighted neuronal spike trains. Weighted spike trains for each neuron were determined by multiplying the spike train of each neuron by the eigenvalue weights of each PC. Line and scatterplots of eigenvalue weights were created to assess changes in neural relationships before, during, and after LC stimulation. PSTHs were generated from PC eigenfunctions of the spike trains of individual recorded units (Chapin and Nicolelis, 1999; Devilbiss and Waterhouse, 2002). The averaged pretreatment values of each group were compared with the corresponding responses during LC stimulation conditions, and analyzed via a two-way ANOVA with repeated measures. Dunnett's post hoc tests were used to determine significant differences between pretreatment and LC stimulation conditions.

Videotape analysis of the entire experimental session with a video counter timer providing time stamps (resolution, $0.1 \mathrm{~s}$ ) synchronized to the multiunit recording and stimulus control systems provided a means to confirm that animals remained in a quiet resting state throughout the experiment. A quiet resting state in these experiments was defined behaviorally as an animal lying down with its head raised from the chamber floor.

Microdialysis. Microdialysis experiments began after a stable baseline of three samples was achieved. After initial baseline measurements, the LC was stimulated at $300 \mu \mathrm{A}$ at $1.0 \mathrm{~Hz}$. Statistical differences between baseline conditions and LC stimulation conditions were determined with a paired $t$ test. After return of NE levels to baseline levels, the series of control and tonic LC stimulation conditions used for electrophysiological experiments were delivered. Each 20 min dialysate sample was matched to one of the control, recovery, or four tonic LC stimulation conditions. Once NE levels returned to baseline, NE determined from randomized LC stimulation conditions was not tested for statistical significance because of possible sample to sample interference.

Histology. Electrode placement within the VPM thalamus, ipsilateral LC, and contralateral whiskerpad was verified by passing $60 \mu \mathrm{A}$ of current across two microwires for $45 \mathrm{~s}$, and then perfusing with $0.9 \%$ saline followed by a $10 \%$ formalin solution containing $5 \%$ potassium ferrocyanide which produces a Prussian blue reaction product. Verification of the placement of LC electrodes was important for establishing the anatomical specificity of LC-mediated effects.

\section{Results}

\section{Effects of tonic LC stimulation on LC-NE output}

Tonic electrical activation of the LC over a range of low-intensity, low-frequency stimulus conditions produced increases in NE release within the VPM thalamus as measured by microdialysis/ HPLC-EC methods. Significant increases (paired $t$ test, $p=$ 0.0104) in thalamic NE levels from initial baseline samples ( $1.56 \pm 0.30 \mathrm{pg} / 15 \mu \mathrm{l} ; n=7$ animals) were observed during 1.0 $\mathrm{Hz}, 300 \mu \mathrm{A}$ tonic LC stimulation (Fig. $1 A, B$ ). After cessation of LC stimulation, NE levels in dialysate samples returned to baseline within the following sampling period. Furthermore, after re-establishing baseline levels $(1.37 \pm 0.22 \mathrm{pg} / 15 \mu \mathrm{l})$, a linear increase in thalamic NE efflux was observed with increasing fre- 
quencies of tonic LC stimulation $(0.5-5.0 \mathrm{~Hz}$ at $100 \mu \mathrm{A})$. Increases in NE release were only observed when LC stimulation electrodes were placed within or juxtaposed to the core of the LC nucleus (Fig. 1C). Generally, tonic stimulation increased the discharge rate of LC neurons in an additive manner (Fig. 1D). For example, for this animal, $1.0 \mathrm{~Hz}$ LC stimulation increased tonic discharge in the quietly resting rat from 1.5 to $3.1 \mathrm{~Hz}$. However, across all animals tested, increasing frequencies of LC stimulation (Stim) increased LC discharge from $0.85 \mathrm{~Hz}$ (baseline/control) to $0.90(0.5 \mathrm{~Hz}$ LC Stim) to 1.33 (1.0 Hz LC Stim) and 3.15 (5.0 Hz LC Stim). Together, these observations indicate that LC stimulation through microwire electrode bundles with currents 5- to 10 -fold lower than those used previously produced activation of LC neurons and increases in VPM thalamic NE that were comparable with previously reported levels (Florin-Lechner et al., 1996; Berridge and Abercrombie, 1999).

Effects of increasing frequencies of tonic LC stimulation on responses of VPM thalamic neurons to sensory-driven input The effects of LC activation on neuronal responsiveness to afferent input were examined in 191 units recorded from the VPM thalamus of six awake, quietly resting animals. Only units that met strict off-line verification procedures were classified as single neurons and included in these analyses (Fig. 2A, black unit). Accordingly, 74 of the 191 recorded units were verified as single neurons, 40 of which responded to whiskerpad stimulation. The magnitude of the sensory-evoked responses from these cells was altered during tonic LC stimulation conditions that produced significant increases of NE within the VPM (Fig. $2 B$ ). When stimulating electrodes were placed outside $(>250 \mu \mathrm{m})$ the LC, no effect or variable results were observed (Devilbiss and Waterhouse, 2004) and were not considered further in this study. Overall, thalamic sensory-evoked excitatory responses were modulated according to a biphasic or "inverted-U" LC-mediated response function (Fig. $2 C$ ) (ANOVA; $F_{(3,58)}=3.57 ; p=0.015$ ). During initial periods of low-level LC activation, sensory-driven responses were facilitated. With increasing levels of LC stimulation, sensory-driven responses of the VPM thalamus were further enhanced to a peak value (2.15-fold increase above control response levels). Additional increases in LC activation reduced evoked discharge from the peak value yielding the biphasic or inverted-U LC-mediated modulatory response profile.

Although the overall effect of increasing LC-NE output was to elicit an inverted- $U$ modulatory profile for sensory evoked discharge, individual cells expressed distinctive responses to LC stimulation that were independent from one another (Fig. 3). These effects included facilitation (57\%), suppression (23\%), or negligible effect $(20 \%)$ on sensory stimulus-evoked discharges ( $n=40$ whisker responsive neurons). Importantly, these cellspecific LC-mediated modulatory effects were observed across ensembles of simultaneously recorded VPM thalamic neurons. For example, in representative recordings from neighboring VPM thalamic neurons (Fig. 3), facilitation was characterized by either an inverted-U modulatory profile (Fig. $3 A$, cell 1 ) or a continually increasing LC-stimulation frequency response curve (cell 2). In contrast, the suppressant profile of action was characterized by a monotonic response curve, such that stimulusevoked responses were progressively reduced over increasing LC stimulation frequencies (Fig. 3A, cell 3). Thus, consistent with previous studies (Rogawski and Aghajanian, 1980; McCormick and Prince, 1988; George, 1992; Devilbiss and Waterhouse, 2000, 2004), LC activation or NE application can differentially affect
A.
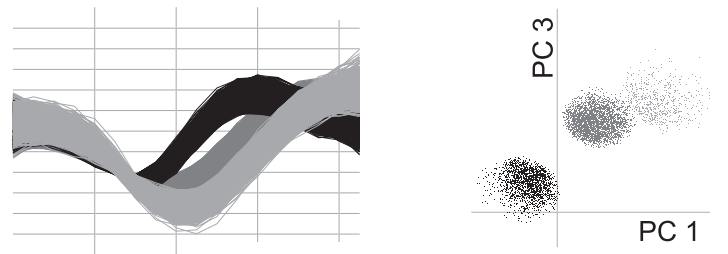

B.

Control $1 \mathrm{~Hz}$ LC Stimulation

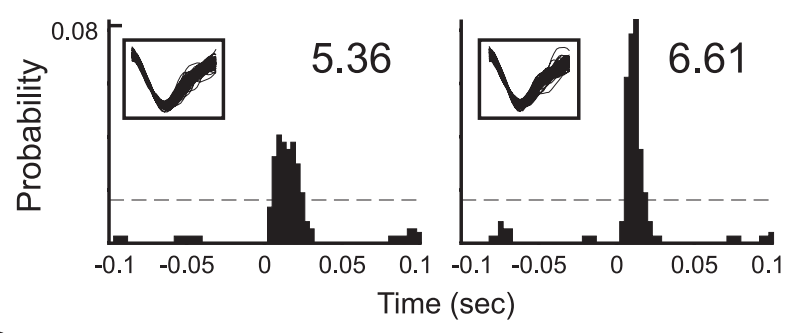

C.

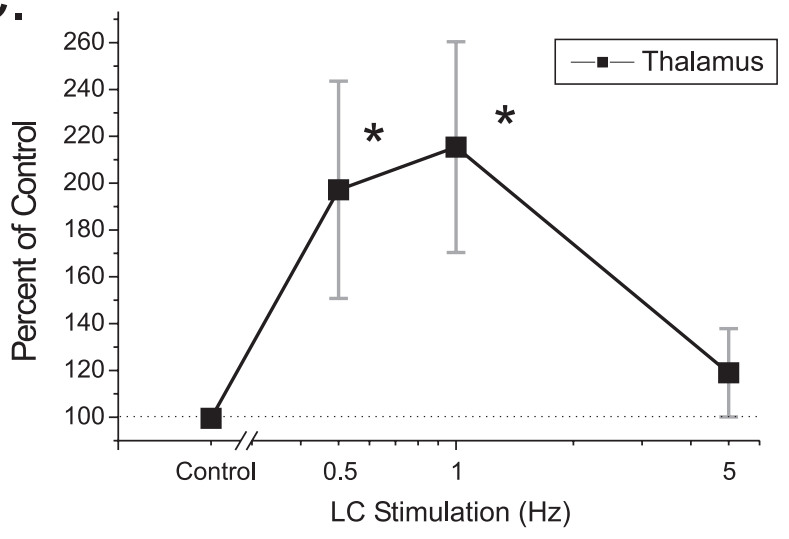

Figure 2. The effect of $L C$ stimulation on individual thalamic neuron responses to whiskerpad stimulation. A, Representative discrimination of waveforms recorded simultaneously from rat VPM thalamus. The single unit (black traces) verified by off-line analysis was characterized by visually distinct waveform patterns (left) that were spatially isolated from other waveform clusters when visualized in principal component space (right). B, Peristimulus time histograms demonstrate somatosensory stimulus-evoked discharge of a typical VPM thalamic neuron before (left) and during $1.0 \mathrm{~Hz} \mathrm{LC}$ stimulation (right). Inset numbers represent the summed probability of neuronal discharge during the excitatory response. For each histogram, the vertical axis represents the probability of neuronal discharge during a given time bin ( $x$-axis). Whiskerpad stimulation was presented at $0.0 \mathrm{~s}$. The inset waveforms indicate the stability of the cell recording across experimental time. $C$, Average change in stimulus-evoked response for all recorded VPM thalamic neurons $(n=40)$ over the range of tonic $\mathrm{LC}$ stimulation frequencies. The maximal, statistically significant increase in VPM thalamic neuronal responsiveness to synaptic input was observed at $1.0 \mathrm{~Hz}$ tonic $\mathrm{LC}$ stimulation $\left({ }^{*} p<0.05\right)$. Error bars indicate SE.

the responsiveness of thalamic neurons responding to the same peripheral stimulus.

The optimal LC output for eliciting peak responses to stimulus-driven synaptic input was specific for each thalamic neuron. For example, the stimulus-evoked discharge of cell 1 (Fig. $3 B$ ) was maximally enhanced (1.6-fold from control) during $1.0 \mathrm{~Hz}$ LC stimulation, whereas cell 2 demonstrated a maximal 2.1-fold facilitation during $5.0 \mathrm{~Hz}$ stimulation. Thus, although an optimal level of LC output for enhancing VPM thalamic neuron responsiveness to synaptic input could be identified, the level of LC activation for achieving this effect was often different for neighboring cells in the same animal. An optimal level of LC 
A.

B.
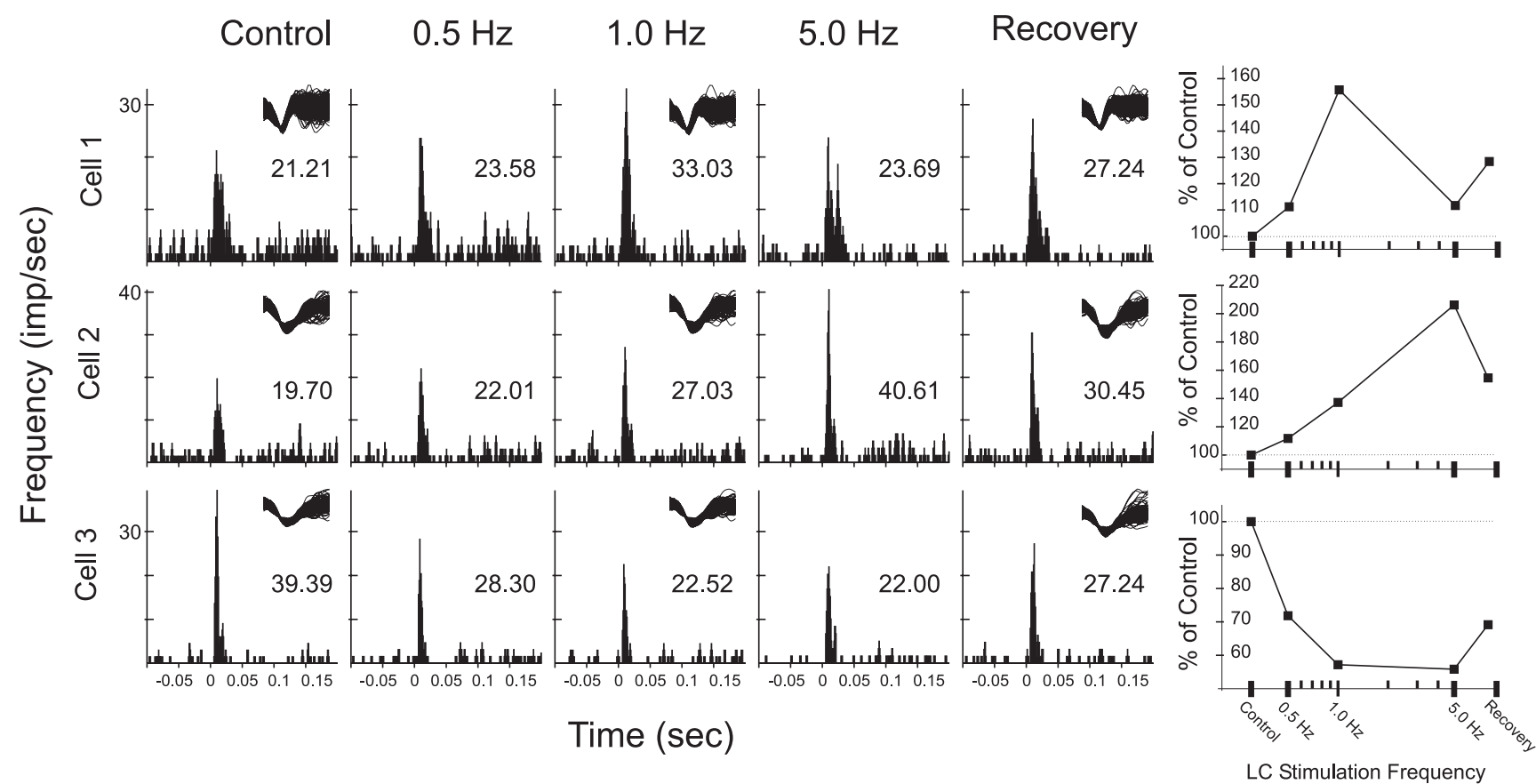

Figure 3. The effects of tonic LC output on somatosensory-evoked discharge of simultaneously recorded thalamic neurons. Peristimulus time histograms illustrate sensory-driven responses of three simultaneously recorded VPM thalamic neurons ( 1 each row) before (left column), during (middle; $0.5-5.0 \mathrm{~Hz}$ ), and after (right column) periods of $\mathrm{L} C$ stimulation. Whiskerpad stimulus-evoked excitatory discharges were facilitated according to a parabolic/inverted-U relationship (cells 1 and 2 ) or were monotonically suppressed (cell 3 ) with increasing $L C$ stimulus frequencies. For each histogram, the vertical axis represents the frequency of discharge for a given time bin. The horizontal axis represents time before and after the onset of the stimulus presentation ( $0 \mathrm{~s}$ ). All histograms sum the unit activity during an equivalent number of stimulus trials. Each numerical inset indicates the peak response frequency of the response of the cell to whiskerpad stimulation. The inset waveforms indicate the stability of individual neuron recording across experimental time. $\boldsymbol{B}$, Line graphs indicate the peak stimulus-evoked excitatory discharge for each experimental condition (percent of control). Note that cell 2 required a higher frequency of $L C$ stimulation than cell 1 to achieve its peak facilitated response.

output was also observed for neurons exhibiting suppression of sensory-evoked responses with increasing LC output. For example, cell 3 (Fig. $3 B$ ) responded most vigorously under control conditions of basal LC discharge rates. However, with increasing LC output sensory-evoked responses were monotonically suppressed to a maximal 1.7-fold suppression during $5.0 \mathrm{~Hz}$ LC stimulation conditions. Thus, for this neuron, the optimal response to sensory input was observed at baseline levels of tonic LC output.

\section{Population level analysis: cross-correlegrams}

To test the hypothesis that LC output modulates the representation of sensory signal coding across ensembles of thalamic cells, we examined the effect of LC activation on correlated or nearcoincident discharge between simultaneously recorded neurons (Gerstein et al., 1989). As illustrated by the cross-correlegram shown in Figure 4, many neuron pairings demonstrated a significant relationship between their spike train discharges. After appropriate corrections for direct sensory-stimulus effects, the correlegram for this representative pair indicates a temporal relationship whereby the reference neuron tends to discharge 20 ms before the paired second neuron. Such a temporal relationship indicates a functional, although not necessarily a direct, synaptic connection between these two cells (Aertsen and Gerstein, 1985; Aertsen et al., 1989; Devilbiss and Waterhouse, 2002). To extend this analysis across all neuronal pairs in a recorded ensemble, these relationships were visualized by plotting incidences of significant correlation [exceeding 99\% confidence intervals (CIs)] as a scattergram (Fig. 5A) for each condition. Moreover, the probability of correlated discharge between each neuronal

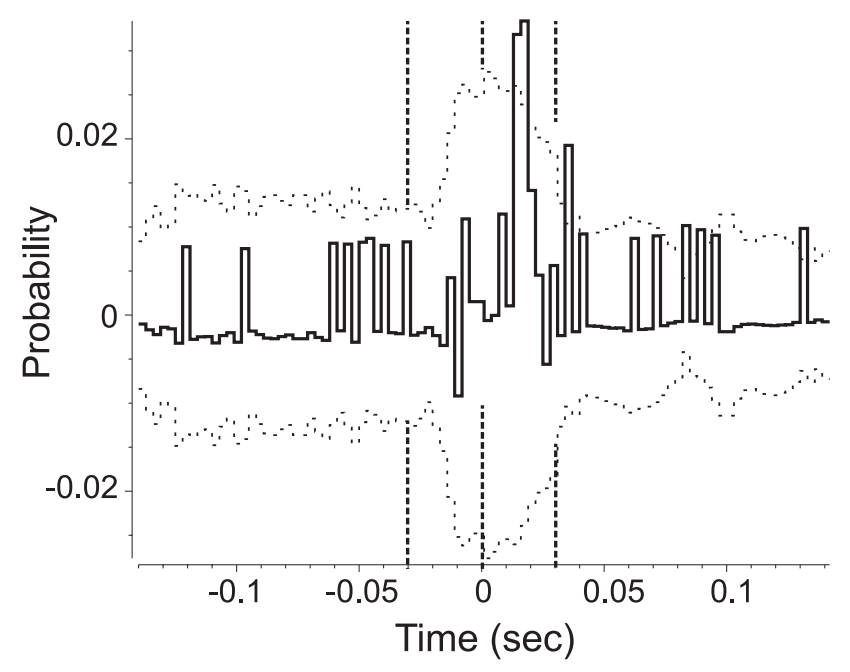

Figure 4. Representative cross-correlegram between two VPM thalamic neurons. During control conditions, significant correlation between spike trains $(+13-18 \mathrm{~ms})$ of this neuron pair indicates that they are functionally connected. Additionally, the latency of the correlegram peak indicates that this cell preferentially discharges after the reference neuron. Correlated discharge between these neurons was subtracted from a PSTH shift-predictor and plotted (solid line) in conjunction with $99 \%$ confidence intervals for the correlegram (dotted line). The $y$-axis represents the probability of correlation; the $x$-axis depicts time before and after thalamic reference neuron discharge ( $0 \mathrm{~ms}$ indicated by center vertical line). Correlations between $\pm 30 \mathrm{~ms}$ (indicated by outer vertical lines) were further analyzed. 
Ai. (Control)

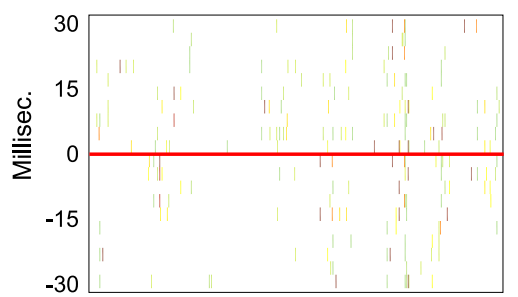

Aiii. $(1.0 \mathrm{~Hz})$

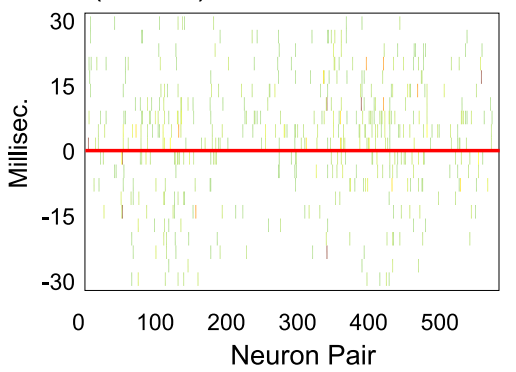

B.

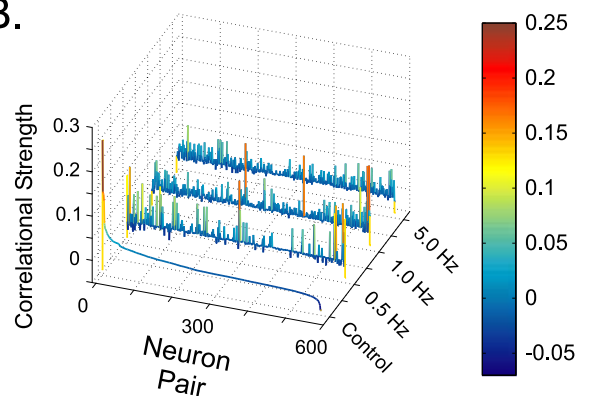

Figure 5. Effects of $L C$ activation on functional connectivity within an ensemble of VPM neurons. Ai-Aiv, Functional connectivity, defined by patterns of correlational strength, was plotted before (Ai) and during (Aii-Aiv) periods of $\mathrm{L} C$ tonic stimulation. Significant ( $\pm 99 \% \mathrm{Cls}$ ) incidences of correlation were plotted for each combination of neuron pairs ( $x$-axis) before and after the reference spike ( $y$-axis) for this representative case. Strength of significant correlations was indicated by the tick color (color bar). Increasing levels of $\mathrm{LC}$ stimulation $[0.5 \mathrm{~Hz}$ (Aii), $1.0 \mathrm{~Hz}$ (Aiii), $5.0 \mathrm{~Hz}$ (Aiv)] produced an inverted-U response profile with respect to the number of significant correlations. $\boldsymbol{B}, A$ waterfall plot of rank-ordered data from $\mathbf{A i}$-Aiv demonstrates that LC-induced pairs were rank-ordered according to correlational strength during control ( $x$-axis, neuron pair; $y$-axis, correlation strength; $z$-axis, LC stimulation condition). Increasing LC output levels $(0.5,1.0$, and $5.0 \mathrm{~Hz})$ increased or decreased neuron pair correlational strength regardless of rank-order position. $C$, Graphic representation of average changes in correlation strength. Across all animals tested $(n=6)$, the number and strength of significant correlations between neurons in recorded ensembles changed as a function of $\mathrm{LC}$ stimulation frequency. The relationship between the number of correlated pairs and correlation strength and $\mathrm{LC}$ output followed an inverted-U response profile. These data indicate that increasing $\mathrm{LC}$ output dynamically reconfigures the functional circuitry between VPM neurons engaged in coding of sensory stimuli. ${ }^{*} p<0.05 ;{ }^{* *} p<0.001$. Error bars indicate SE. changes in the pattern or strength of correlations between neurons were not dependent on initial correlational strength. Neuronal

relations between neurons. For example, the number of significant correlations during $0.5 \mathrm{~Hz}$ stimulation (Fig. 5Aii, 249/ 12,096) did not change substantially from control conditions (Fig. Ai, 308/12,096). However, the number of significant correlations was increased 1.85 -fold from control during $1.0 \mathrm{~Hz}$ stimulation (Fig. 5Aiii, 569/12,096). Additional increases in LC stimulation produced a reduction in the number of correlation incidents from the peak value (Fig. 5Aiv) but one that was still higher than control, thus yielding a biphasic or inverted-U relationship for this index of LC action. Importantly, for a given subject, the number of significant correlations did not change across control epochs within an experimental session (data not shown).

Additional examination of the effects of LC activation on correlated activity between VPM thalamic neurons demonstrated that correlation strength (absolute value of probability of near-coincident discharge) between neuron pairs was also modulated across an inverted-U modulatory profile. These effects were independent of the above changes in the number of significant correlations. For the representative case in Figure 5, 0.5 Hz LC stimulation elicited an increase in correlational strength for 540 neuron pairings (Fig. 5 Aii). Additional increases in LC output (1.0 Hz stimulation) yielded 845 instances of increased correlation strength (Fig. 5Aiii). However, the highest level of LC output $(5.0 \mathrm{~Hz})$ tested produced fewer ( $n=777)$ instances of increased correlation strength. These results, combined with the above changes in the number of significant correlations, suggest that increasing LC output increases the number and strength of functional connections among neurons within the VPM thalamus according to an inverted- $U$ response relationship.

pair was indicated by a color-coded scale. During control and LC-stimulation conditions, significant correlations were observed typically between $30 \mathrm{~ms}$ before and $30 \mathrm{~ms}$ after the discharge of the reference neuron. Additionally, after confirming that a single neuron was not recorded on multiple electrodes (see Materials and Methods), correlations were observed both within and across microwire electrodes.

Tonic LC stimulation produced several distinct effects on correlated activity within an ensemble of neurons (Fig. $5 A-C$ ). First, increasing levels of LC output incrementally altered the pattern of functional connectivity. These effects are indicated in Figure $5 \mathrm{~A}$ as changes in the pattern of significant correlations between neuron pairs. For example, a given neuronal pair of the ensemble either demonstrated correlated activity during control conditions (Fig. 5Ai) and no correlated activity during LC stimulation (Fig. 5Aii) or vice versa. Second, increasing LC output (Fig. 5Aii$i, A i v)$ led to increases in the absolute number of significant cor-
Despite the overall increase in correlation strength with increasing LC output, we noted that initially strong correlations (positive or negative) observed during control conditions tended to be reduced with increasing LC output. This effect is depicted in Figure $5 A i-A i v$ as a reduction in the number of red and blue (corresponding to strong positive or negative correlations, respectively) points. Thus, these data were furthered examined to test whether LC induced changes in correlation strength may be dependent on the initial strength of correlation. Average correlational strength over $20 \mathrm{~ms}( \pm 10 \mathrm{~ms}$ around the reference spike) for each neuron pair (Fig. 5A) was rank-ordered for control conditions. Retaining rank order, the average correlation strength for each neuron pair was plotted for control and LC stimulation conditions (Fig. 5B). During 0.5 and $1.0 \mathrm{~Hz}$ LC stimulation, the correlation between spike trains was increased for several neuron pairs that initially demonstrated negative correlations (right side of waterfall plot), thus reducing the strength of their negative 
correlation. In contrast, the correlation between strong positively correlated neuronal pairs was diminished with increasing LC stimulation (left side of plot). Additionally, weakly correlated neuronal pairs (center of plot) demonstrated a heterogeneous mix of increases and decreases in correlational strength with increasing frequencies of LC stimulation. Thus, the net effect of LC activation was to reduce the extreme positive and negative correlations among cells within the recorded ensemble, while increasing the overall functional connectivity within the recorded array.

The above analyses used to determine changes in correlated discharge between neurons of the VPM thalamus were performed on data sets from all subjects yielding similar results. As such, activation of the LC efferent path elicited similar effects on functional connectivity across all animals tested (Fig. $5 C$ ). Both the number (ANOVA; $\left.F_{(3,26)}=13.91 ; p<0.0001\right)$ and strength (ANOVA; $\left.F_{(3,26)}=6.04 ; p=0.0028\right)$ of significantly correlated discharge patterns were modulated from the control condition with increasing levels of LC stimulation according to an inverted-U response relationship. These data indicate that functional connectivity, as defined by patterns of correlated spike train discharge, is altered in the VPM thalamus with increasing levels of LC activation.

\section{Population level analysis: PCA eigenvalue weights}

Distributed patterns of stimulus-related discharge within the VPM thalamus were further studied with PC analysis. Previous studies in vibrissa-related regions of the VPM thalamus (i.e., barreloids) have demonstrated that eigenvectors (vectors of PC weights) derived from PC analysis of multineuron spike train activity are conserved across animals and represent specific stimulus properties (e.g., direction of vibrissa movement) (Chapin and Nicolelis, 1999). In the current study, functionally relevant relationships between recorded neurons were determined when PC eigenvalues were rank ordered according to latency and magnitude of their sensory evoked responses (Fig. 6). Individual VPM thalamic neurons exhibit the strongest response with the shortest latency to sensory input from a principal or primary whisker on the contralateral face (Diamond et al., 1992). As illustrated in Figure $6 \mathrm{~A}$, rank-ordering neurons by response characteristics (sum of mean evoked-discharge and response latency) resulted in PC eigenvector patterns that were representative of principal whisker relationships (Chapin and Nicolelis, 1999). Neurons with the largest response and shortest latency to whiskerpad stimulation are positioned on the left in Figure 6, with the weakest response and longest latency placed on the right. The PC eigenvector weights shown in Figure $6 A$ are representative of the patterns found across all animals in this study. Overall, PC1 contained all positive and relatively homogeneous PC weights, whereas PC2 demonstrated a linear increase in eigenvector weights across the ordered array of neurons. As such, the positive, homogeneous weights of PC1 indicate that this component represents generalized neuronal activity across the neural ensemble. In contrast, the linear relationship between the weights of PC2 and neuronal response properties suggests that $\mathrm{PC} 2$ represents a more specific description of sensory information. For example, PC2 likely represents a sensory coding scheme that is dependent on a graded relationship between neuronal activity across an ensemble and a specific dimension of receptive field structure, such as direction of movement across the array of facial vibrissae (Chapin and Nicolelis, 1999).

Additional analysis of $\mathrm{PC}$ weights identified changes in eigenvalues with increasing levels of LC activation. Changes in PC1 weights from control values are illustrated in Figure $6 \mathrm{Bi}$. Neurons

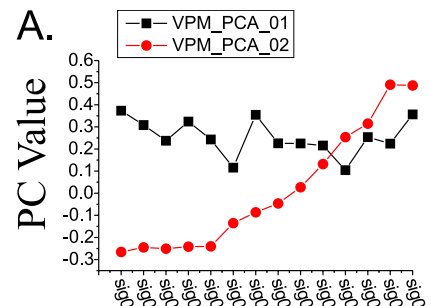

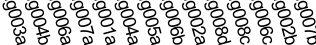
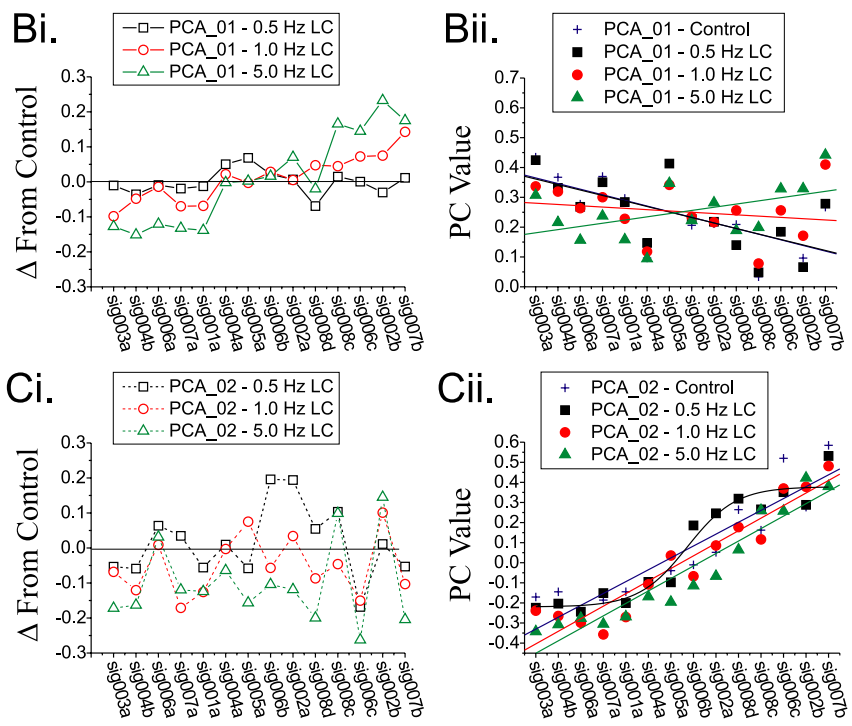

Figure 6. $\quad P C$ weighting coefficients calculated from an array of 16 simultaneously recorded VPM thalamic neurons. $\boldsymbol{A}$, Graph depicting PC eigenvalue weight coefficients 1 and 2 from the ensemble of thalamic neurons during periods of quiet rest $(t=1100 \mathrm{~s}$ ). The neurons on the $x$-axis are rank ordered according to sensory response characteristics (evoked response magnitude by evoked response latency). Shorter latencies and larger responses to sensory stimuli indicate a cell that is receiving input from the center of the receptive field of the neuron. Rank-ordering data by this method yields results similar to those reported previously by Chapin et al. (1999). Bi, Bii, PC1 eigenvalues (Bi) and the corresponding changes from control (Bii) are plotted for each cell during different $\mathrm{LC}$ output conditions (control, $0.5,1.0$, and $5.0 \mathrm{~Hz}$ stimulation). With increasing $\mathrm{LC}$ stimulation, the slope of the linear regression lines are rotated counterclockwise from 0.676 (control) to $0.011(0.5 \mathrm{~Hz})$. Additionally, the coefficient of determination decreased $\left(R^{2}=0.458\right.$, control; $R^{2}=0.231,0.5 \mathrm{~Hz}$ ), indicating greater variation in eigenvalues and, thus, a loss of directional information represented by PC1. $C$, As in $B, P C 2$ eigenvalues ( $\boldsymbol{C}$ ) and the corresponding changes from control (Cii) are plotted for each cell during different $\mathrm{LC}$ output conditions (control, $0.5,1.0$, and $5.0 \mathrm{~Hz}$ stimulation). In this case, the slope of the linear regression lines were not rotated in conjunction with increasing $L C$ stimulation, and the variability of eigenvalues was slightly reduced $\left(R^{2}=0.827\right.$, control; $R^{2}=0.896$, $0.5 \mathrm{~Hz})$. However, $P C 2$ eigenvalues were best fitted with a sigmoidal curve $\left(R^{2}=0.940\right)$ during $0.5 \mathrm{~Hz} \mathrm{LC}$ stimulation. Overall, these data indicate that increasing tonic LC output levels reduce the relationship between neuronal response properties and their contribution to the PC1 and more strongly relate neuronal response properties to $P C 2$. It is likely that these actions result in a greater discrimination of sensory signals at low levels of LC output and a less variable (i.e., more discrete) representation at higher levels.

that were plotted at the extremes of the rank-ordering scale demonstrated the largest changes in PC weights during increasing LC activation. These changes in $\mathrm{PC}$ weights resulted in rotation of the slope of PC1, such that with increasing levels of LC stimulation the slope of PC1 became equivalent to zero (Fig. 6 Bii). For example, during control conditions, the slope of the regression line representing PC1 weights was significantly different from zero (slope, $-0.0189 ; R^{2}=0.4567 ; F_{(3,51)}=10.12 ; p=0.079$ ). However with increasing LC stimulation, a significant positive rotation of this regression line was observed $\left(f_{(x)}=-0.01842 \times\right.$ $x+0.3798,0.5 \mathrm{~Hz} ; f_{(x)}=-0.004311 \times x+0.2845,1.0 \mathrm{~Hz} ; f_{(x)}=$ $\left.0.01069 \times x-0.001586,5.0 \mathrm{~Hz} ; F_{(3,48)}=5.59735 ; p=0.0023\right)$. 
In other words, in a frequency-dependent manner, LC stimulation decreased the contribution of both robustly responding and weakly responding neurons (e.g., sig004b and sig002b) (Fig. 6 Bi) to $\mathrm{PC} 1$, thus minimizing the relationship between neural rank order and PC weight (Fig. 6ii). Additionally, with increasing levels of LC stimulation, the coefficient of determination $\left(R^{2}\right)$ of the linear regression was reduced $(0.5 \mathrm{~Hz}, 0.4331 ; 1.0 \mathrm{~Hz}, 0.04072$; $5.0 \mathrm{~Hz}, 0.2308)$. Reductions in $R^{2}$ and fit of the data to the linear model further supports the position that the relationship between neural rank order and PC1 weight is reduced with increasing LC output.

In contrast to LC-mediated effects on PC1, the slope of PC2 weights (Fig. 6Ci,Cii) across rank-ordered neurons was not changed significantly with increasing levels of LC activation. Slopes of PC2 remained parallel but had significantly different $y$-intercepts (Fig. 6Ci) (control, $-0.3893 ; 0.5 \mathrm{~Hz},-0.3823 ; 1.0$ $\left.\mathrm{Hz},-0.4665 ; 5.0 \mathrm{~Hz},-0.5127 ; F_{(3,51)}=4.3288 ; p=0.00857\right)$. Likewise, the coefficient of determination $\left(R^{2}\right)$ was increased rather than reduced with increasing levels of LC stimulation (control, 0.8272; $0.5 \mathrm{~Hz}, 0.8959 ; 1.0 \mathrm{~Hz}, 0.8931 ; 5.0 \mathrm{~Hz}, 0.9053$ ), indicating a better fit of the data to the regression line with increasing activation of the LC efferent path. However, it is worth noting that during $0.5 \mathrm{~Hz}$ LC stimulation (not others), the eigenvalues of several neurons from this case were increased from control values (Fig. 6Cii, sig006b-sig008c). These increases altered the eigenvalue pattern and were best fitted with a nonlinear, sigmoidal curve $\left(R^{2}=0.940\right.$ vs $R^{2}=0.896$ for linear regression). The improved fit of PC2 weights with a sigmoidal curve indicates that the gradient of PC2 weights across the ensemble of neurons began to approach a step function. As such, the effects of low levels of LC output $(0.5 \mathrm{~Hz})$ on the contribution of VPM neuron discharge patterns to PC2 putatively reflect more clearly defined center/surround delineation across the receptive field. By association, this effect suggests that the relation of each neuron's discharge pattern to whisker movement was more clearly defined at this level of LC output. With additional increases in LC activation $(5.0 \mathrm{~Hz})$, PC2 weights across rank-ordered neurons were again best fitted with the linear regression model.

In addition to the relationship between PC eigenvalues and the latency and magnitude of sensory evoked responses of each neuron, the percentage total variance of neuronal firing patterns explained by each PC was determined. PC1 exhibited a modest inverted-U response profile with respect to LC output levels. The percentage of variance explained by PC1 peaked at $1.0 \mathrm{~Hz} \mathrm{LC}$ stimulation (2.4\% above control) and fell below control levels (97.6\% of control) with additional increases in LC output. This increase at $1.0 \mathrm{~Hz} \mathrm{LC}$ stimulation suggests that more variability of the neuronal spike train (i.e., responses) is allocated to represent the overall (global) response. In contrast to the modest changes in $\mathrm{PC} 1$, the percentage total variance of neuronal firing patterns explained by PC2 successively increased to $12 \%$ above control conditions with increasing levels of LC output (control, 6.656\% vs $5.0 \mathrm{~Hz}, 7.425 \%$ ). The more pronounced increase in variance explained by PC2 suggests that at higher levels of LC activation, more of the variability within neuronal spike train activities (i.e., responses) is allocated to the representation of the sensory signals distributed across the neuronal ensemble. For example, PC2 and its relationship to direction of whisker movement may be more robustly coded in the distributed activity of ensembles of neurons.

In summary, increasing levels of LC activation produced significant effects on the PC1-PC2 weights for ensembles of sensory thalamic neurons. The actions of increasing LC stimulation re-
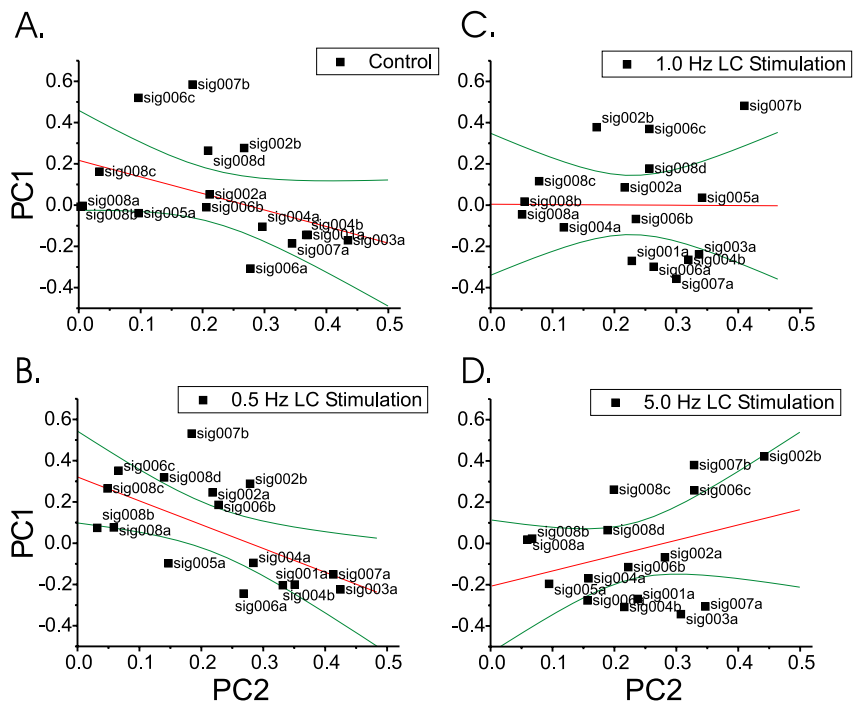

Figure 7. The effect of $L C$ stimulation on functional relationships between neurons. For each of 16 simultaneously recorded VPM neurons, the $P C$ eigenvalue weighting coefficient of the first $P C(P C 1 ; x$-axis) was plotted against the second ( $P(2 ; y$-axis). The result is a graphic representation of the relationships between neurons within the recorded array. These plots were created for control $(\boldsymbol{A})$ and $L C$ stimulation $[0.5 \mathrm{~Hz}(\boldsymbol{B}), 1.0 \mathrm{~Hz}(\boldsymbol{C}), 5.0 \mathrm{~Hz}(\boldsymbol{D})]$ periods. For each level of $\mathrm{LC}$ activation, neuron position changes within the space defined by $P C 1$ and $P C 2$ and a shift in the linear regression slope indicate changes in the contribution of each neuron to these dimensions of stimulus information processing.

sulted in a more variable set of PC1 weights that were less representative of stimulus properties than during control conditions. Therefore, as LC activation increased, the relatively nonspecific nature of PC1 with respect to stimulus attributes became more pronounced. In contrast, with increasing LC stimulation, the shape of the regression for PC2 weights shifted from a linear to a sigmoidal representation. This shift suggested that the relationship between individual neuron discharge patterns and stimulus properties was more clearly defined during periods of elevated LC output. Furthermore, at the highest level of LC stimulation, more of the combined activity across this ensemble of neurons was dedicated to the representation of higher-order coding of whiskerpad stimulation.

\section{Population level analysis: PCA eigenvalue weight maps}

The weights for each PC are, in essence, coordinates that map the discharge pattern of each neuron within the multidimensional PC space. Thus, weights for each PC represent a definition of the role of each neuron in processing multidimensional sensory information. PC1 and PC2 dimensions of sensory information processing are hypothesized to encode magnitude and direction of whisker deflection. Increasing levels of LC stimulation altered the position of each neuron within the first two dimensions of PC space (i.e., PC1 vs PC2). For example, the weights for PC1 and PC2 for an ensemble of 16 simultaneously recorded VPM neurons are plotted in Figure 7. Across conditions of increasing LC activation, the location of each neuron in PC space changes. Low levels of LC stimulation produced a more negative slope of the regression line for these data (control, -0.8019 vs $0.5 \mathrm{~Hz}$, $-1.1539)$, which was significantly different from the other slopes $(F=7.728 ; p=0.0148)$. Additional increases in LC activation rotated the slope in a positive direction (maximal positive slope, $0.74365 .0 \mathrm{~Hz}$ ). However, the neuronal representations in PC space did not rotate as a whole cluster, but independently moved within the PC space with increasing frequencies of LC stimula- 
tion. These data further indicate that output from LC is capable of altering the contribution of a neuron to different dimensions of sensory information processing.

\section{Population level analysis: PCA eigenfunctions}

$\mathrm{PC}$ weights in conjunction with the neuronal spike-train data from simultaneously recorded single neurons of the VPM within individual animals were used to construct a number of eigenfunctions. Specifically, eigenfunctions were calculated as the principal component eigenvector multiplied by the spike-train activity of a single neuron.

As illustrated in Figure $8 A-B$, the first $P C$ eigenfunction exhibited an inverted- $U$ response curve across the range of tonic LC outputs tested $\left(F_{(4,19)}=23.4 ; p<\right.$ $0.0001)$. The maximal increase in magnitude (1.16-fold) was observed during 1.0 $\mathrm{Hz}$ LC stimulation. Likewise, the second PC eigenfunction, demonstrated an inverted- $U$ response curve across the range of LC outputs tested $\left(F_{(4,19)}=5.46\right.$; $p<0.0097)$, but the maximal increase in PC2 was substantially larger than PC1 (2.57-fold). Additional increases in LC activation $(5.0 \mathrm{~Hz})$ suppressed the augmented responses of PC1 and PC2 toward control levels and below. As indicated above, the magnitude of nonstimulus related (background) activity of PC1 and PC2 was also modulated by increasing levels of LC stimulation. Background activity of PC1 and PC2 was incrementally suppressed with increasing LC stimulation to a maximum during $1.0 \mathrm{~Hz} \mathrm{LC}$ stimulation (PC1, $69 \%$ of control at $0.5 \mathrm{~Hz}, 27 \%$ at $1.0 \mathrm{~Hz} ; \mathrm{PC} 2$, $49 \%$ at $0.5 \mathrm{~Hz}, 51 \%$ at $1.0 \mathrm{~Hz}$ ). The suppression of background activity in PC1 and PC2 was minimal (near control levels) with additional increases in LC activation $(5.0 \mathrm{~Hz})$.

This series of PCA analyses demonstrated for this representative animal were performed on data sets from all subjects yielding similar results. The effects of increasing LC stimulation on population coding schemes (represented by PC1 and PC2 in this analysis) were observed across all animals tested (Fig. 8 C). Overall, increasing the frequency of tonic LC stimulation elicited a parabolic or inverted- $U$ stimulus-response curve for PC1 $\left(\mathrm{ANOVA} ; F_{(4,52)}=5.70 ; p=0.0011\right)$ and PC2 $\left(\operatorname{ANOVA} ; F_{(4,40)}=\right.$ $3.85 ; p=0.0134)$, as recorded from ensembles of thalamic neurons in multiple animals $(n=6)$. However, across subjects, PC2 was facilitated to a greater extent than PC1. These data indicate that the ability of tonic LC output to alter sensory representations within a distributed ensemble of neurons is robust and conserved across animals.

\section{Discussion}

The present study demonstrates that tonic LC output regulates cellular and local network responses to sensory-driven synaptic input in the VPM thalamus of awake, quiet resting animals. Unilateral microstimulation of LC increased both LC unit discharge and NE efflux in the ipsilateral VPM thalamus. Under these same conditions, VPM single-unit responses to whiskerpad stimulation were modulated in a cell-specific manner according to either an inverted-U or monotonic suppressant response profile. At the ensemble level, distributed representations of sensory stimuli, as measured by PCA or functional connectivity between VPM neurons was also increased over an inverted-U profile with increasing LC output. Together, these observations forge an important link between established cellular actions of the LC-NE system and the impact of this pathway on local thalamic network responses to sensory-driven synaptic input. Furthermore, these results reveal new modes of noradrenergic modulation of sensory signal processing in waking animals.

\section{Heterogeneity of LC actions on single neurons}

Increased LC output produced cell-specific modulation of sensory stimulus-evoked thalamic neuron responses similar to that observed previously with continuous, local iontophoretic administration of NE (Foote et al., 1975; Rogawski and Aghajanian, 1980; Waterhouse and Woodward, 1980; Armstrong-James and Fox, 1983; Kossl and Vater, 1989; Waterhouse et al., 1990; McCormick et al., 1991; George, 1992; Holdefer and Jacobs, 1994). Thus, in our view, the heterogeneous nature of LC-mediated neuromodulatory actions in the unanesthetized animal represents innate interactions between noradrenergic and nonnoradrenergic synaptic influences on target cells under physiologic conditions as opposed to experimental differences imposed by one-cell-at-a-time recording procedures. Previous in vitro and in vivo studies have also demonstrated considerable variability with respect to neuronal sensitivity to NE (Sato and Kayama, 1983; Snow et al., 1999; Devilbiss and Waterhouse, 2000, 2004). These studies demonstrated that "facilitation" was predominantly mediated by $\alpha_{1}$ receptor, whereas "suppression” was likely mediated through NE activation of either the postsynaptic $\alpha_{2}$ or $\beta$ receptor (Devilbiss and Waterhouse, 2000). Thus, the cellular complement of adrenergic receptor subtypes, receptor sensitivity 
for NE binding, and local concentrations of NE are important determinants of LC action on target neurons. Moreover, LC-NE output cannot simply be defined in terms of its ability to upregulate or downregulate stimulus-evoked responses of single neurons, but rather in terms of its ability to alter the repertoire of available responses from an assembly of neurons that encode information across a distributed network. Within this context, it is noteworthy that distributed activity across ensembles of neurons is believed to be critical for accurate coding of sensory information within sensory pathways (Nicolelis et al., 1995; Laurent et al., 1996; Chapin and Nicolelis, 1999; Devilbiss and Waterhouse, 2002).

\section{Dynamic reorganization of the neural ensemble}

The current analyses indicate that the functional connections between VPM neurons were simultaneously strengthened and reduced with increasing LC output, thus resulting in continual changes in connectivity patterns among these cells. Moreover, the size of cell assemblies actively encoding sensory stimuli were expanded and reduced in conjunction with increases/decreases in functional connectivity. Correlated neuronal activity peaks were narrow, suggesting common input or reciprocal connectivity (Csicsvari et al., 1998; Lampl et al., 1999; Marshall et al., 2002; Bartho et al., 2004). Importantly, correlegram peaks were not stimulus induced, given that PSTH corrections were applied to all correlegrams (Gerstein et al., 1989). Moreover, convergent projections from either the principal (PrV) or spinal trigeminal nucleus $(\mathrm{SpV})$ likely provide singular inputs to VPM neurons. For example, both anatomical and electrophysiological studies reveal $\mathrm{SpV}$ neurons have large receptive fields and project to PrV and VPM thalamus, thus eliciting responses in VPM neurons from several whiskers (Chiaia et al., 1991; Wang and Ohara, 1993; Friedberg et al., 2004; Timofeeva et al., 2004). Additionally, differences in response latencies between $\mathrm{PrV}(\sim 6.7 \mathrm{~ms})$ or $\mathrm{SpV}$ $(\sim 11 \mathrm{~ms})$ and VPM neurons (Friedberg et al., 2004) could indeed produce the sharp and delayed peaks that were evident in many cross-correlegrams. Nonetheless, other putative circuit mechanisms could produce correlated discharge between VPM neurons (see below) (Bal et al., 1995b; Nicolelis et al., 1995; Bal and McCormick, 1996; Ritz and Sejnowski, 1997; Lampl et al., 1999; Singer, 1999; Friedberg et al., 2004; Harris, 2005).

Over the range of tonic LC output frequencies tested, target cell assemblies in the thalamus continuously self-organized (i.e., altered cellular contributions to sensory stimulus coding). Although the observed changes in VPM functional connectivity may not strictly define the neural code used by trigeminal circuitry, they are likely to have a significant impact on the cortical representation of peripheral sensory stimuli. In a functional context, cortical neurons operate on a continuum as temporal integrators and coherence detectors (Rudolph and Destexhe, 2003). Synchronous activity, as observed in the current study, provides a strong afferent drive for cortical neurons (Salinas and Sejnowski, 2000; Sejnowski and Paulsen, 2006). For example, during periods of low LC-NE output $(\sim 0.9-1.33 \mathrm{~Hz}$ at $0.5-1.0 \mathrm{~Hz}$ stimulation, respectively) VPM thalamic neurons demonstrated the highest amount of synchronous/correlated discharge. Thus, levels of LC output, associated with active waking (Aston-Jones and Bloom, 1981), likely increase the flow of sensory information from the thalamus to the cortex and result in optimization of sensory stimulus coding for ongoing state-dependent behavioral tasks.

\section{Potential mechanisms underlying VPM neuromodulation}

Mechanisms underlying changes in VPM functional connectivity and sensory coding across LC output levels are poorly understood. Depolarization of VPM neurons by $\alpha_{1}$-receptor activation (McCormick and Pape, 1990; McCormick, 1992) may raise the probability of action potential generation within a subset of VPM neurons, thereby producing near-synchronous discharge (Azouz, 2005) and increased functional connectivity (Aertsen and Gerstein, 1985). With additional increases in LC-NE output, activation of $\beta$-receptors enhances the hyperpolarizationactivated cation current (McCormick, 1992) of VPM neurons and decrease discharge probability and the likelihood of synchronous discharge.

The above described effects were observed only when stimulating electrodes were located in close proximity to LC (i.e., $<250$ $\mu \mathrm{m})$. Thus, tissue activation in this study was limited to LC and peri-LC regions. Nonetheless, LC projections to other brainstem neuromodulatory nuclei, such as the lateral dorsal tegmental (LDT) nucleus (Jones and Yang, 1985; Hallanger et al., 1987; Jones, 1990), represent indirect pathways through which LC stimulation could influence VPM sensory responses. The brainstem LDT is a principal source of acetylcholine (ACh) to the thalamus (Satoh and Fibiger, 1986). Although ACh has been shown to depolarize VPM neurons (McCormick and Pape, 1988; Bal et al., 1995a), LC-NE inhibits LDT-ACh cells (Grant and Highfield, 1991; Kohlmeier and Reiner, 1999) and facilitates LDT-GABAergic interneurons (Kohlmeier and Reiner, 1999). Thus, it is unlikely that LC-mediated changes in LDT output could account for the experimental outcomes observed in the present study.

Actions of NE on nucleus reticularis thalami (nRT) may also influence VPM neuronal firing. For example, high-frequency bursts of action potentials in nRT cells (observed during sleep) lead to rebound EPSPs and discharge of VPM neurons (Bal et al., 1995a). However, NE depolarizes GABAergic nRT neurons (McCormick and Wang, 1991), thereby reducing oscillatory discharge within nRT and the VPM (Lee and McCormick, 1996). Thus, NE actions that suppress low-frequency oscillations of nRT/VPM neurons are unlikely to account for increased VPMcorrelated activity as observed in the current study. On the other hand, noradrenergically mediated suppression of nRT could facilitate evoked discharge within the VPM. Existing evidence suggests that such effects would emerge in transitions from sleep to waking, but the consequences of wake-related increases in NE on nRT neuronal discharge and subsequent effects on VPM neuronal responsiveness is unknown.

Finally, initial evidence suggests that thalamic cell correlations are dependent on cortical thalamic feedback (Sillito et al., 1994). Corticothalamic glutamate release onto VPM mGlu receptors could slowly depolarize and activate VPM neurons (McCormick and von Krosigk, 1992). However, existing evidence indicates that NE depresses activity in cortical-VPM and cortical-nRT pathways (Castro-Alamancos and Calcagnotto, 2001).

In summary, given the range of possible mechanisms, the most parsimonious explanation for the observed modulatory actions is that LC-NE output exerts direct effects on VPM neurons so as to promote synchronous discharge in this sensory relay nucleus during periods of active information gathering.

\section{Relationship between tonic LC stimulation, NE efflux, and sensory signal modulation}

In the current study, LC neurons displayed basal firing rates similar to those reported previously for quietly resting, but awake 
animals $(\sim 0.8 \mathrm{~Hz})$ (Aston-Jones and Bloom, 1981). Electrical stimulation $(0.5-5.0 \mathrm{~Hz})$ of the nucleus increased NE efflux in VPM and elevated the discharge of LC neurons to levels $(\sim 0.9-$ $3.15 \mathrm{~Hz}$ ) observed during active waking (i.e., exploration, orienting) (Aston-Jones and Bloom, 1981). Thus, we believe the results reported here are most apropos to understanding processes expressed in the alert and active animal. The fact that the range of LC discharge in our behaving animals was slightly narrower than reported previously (Aston-Jones and Bloom, 1981) is likely attributable to the fact that our animals were well habituated to their testing environment.

The results of the present study indicate that minimal LC activation $(\sim 0.5-1.0 \mathrm{~Hz})$ and NE output $(15-30 \%)$ are sufficient to optimize individual neuron response properties, connectivity, and distributed representations of afferent information within the VPM thalamus. Additional increases in LC output (i.e., $>5$ $\mathrm{Hz}$ ) are likely related to alarm situations and stress (Berridge and Waterhouse, 2003). Thus, it is of interest that under conditions associated with gathering of sensory information (i.e., exploration), the LC-NE system operates within a range that maximizes information processing capabilities of cellular assemblies within the sensory thalamus. In contrast, under other behavioral circumstances, such as quiet rest or stress, the output from the LC-NE system appears capable of priming the sensory thalamic circuitry for other computational tasks and sensory signal processing strategies.

\section{Functional relevance of LC alterations in target neuron discharge}

Fluctuations in tonic LC-NE levels are linked to the level of performance in sustained attention tasks (Aston-Jones et al., 1994; Rajkowski et al., 1994; Usher et al., 1999), working memory (Arnsten and Dudley, 2005), and decision-related actions (Ivanova et al., 1997; Clayton et al., 2004; Nieuwenhuis et al., 2005) according to an inverted-U function. Despite variability of effects on individual neurons, the impact of this noradrenergic system on stimulus-evoked responses, correlated patterns of neuronal discharge, and distributed representations of sensory information was characterized by a similar inverted- $U$ modulatory profile across physiologic increases in LC-NE output. Thus, we provide the first evidence that heterogeneous cellular neuromodulatory effects associated with tonic LC output combine at a neuronal ensemble level to produce a singular sensory signal modulatory action that is related to behavior/cognition. Moreover, this work provides a perspective on how a brainstem modulatory system can coordinately regulate signal processing across a population of neurons within the circuitry of an ascending sensory network.

\section{References}

Abercrombie ED, Finlay JM (1991) Monitoring extracellular norepinephrine in brain using in vivo microdialysis and HPLC-EC. In: Microdialysis in the neurosciences (Robinson T, Justice Jr J, eds), pp 253-274. Amsterdam: Elsevier.

Aertsen AM, Gerstein GL (1985) Evaluation of neuronal connectivity: sensitivity of cross-correlation. Brain Res 340:341-354.

Aertsen AM, Gerstein GL, Habib MK, Palm G (1989) Dynamics of neuronal firing correlation: modulation of "effective connectivity". J Neurophysiol 61:900-917.

Ahissar E, Haidarliu S, Zacksenhouse M (1997) Decoding temporally encoded sensory input by cortical oscillations and thalamic phase comparators. Proc Natl Acad Sci USA 94:11633-11638.

Ahissar E, Sosnik R, Haidarliu S (2000) Transformation from temporal to rate coding in a somatosensory thalamocortical pathway. Nature 406:302-306.
Akaike T (1982) Periodic bursting activities of locus coerulleus neurons in the rat. Brain Res 239:629-633.

Armstrong-James M, Fox K (1983) Effects of ionophoresed noradrenaline on the spontaneous activity of neurones in rat primary somatosensory cortex. J Physiol (Lond) 335:427-447.

Armstrong-James M, Fox K, Das-Gupta A (1992) Flow of excitation within rat barrel cortex on striking a single vibrissa. J Neurophysiol 68:1345-1358.

Arnsten AF, Dudley AG (2005) Methylphenidate improves prefrontal cortical cognitive function through alpha2 adrenoceptor and dopamine D1 receptor actions: relevance to therapeutic effects in attention deficit hyperactivity disorder. Behav Brain Funct 1:2.

Aston-Jones G, Bloom FE (1981) Activity of norepinephrine-containing locus ceruleus neurons in behaving rats anticipates fluctuations in the sleepwaking cycle. J Neurosci 1:876-886.

Aston-Jones G, Rajkowski J, Kubiak P, Alexinsky T (1994) Locus ceruleus neurons in monkey are selectively activated by attended cues in a vigilance task. J Neurosci 14:4467-4480.

Azouz R (2005) Dynamic spatiotemporal synaptic integration in cortical neurons: neuronal gain, revisited. J Neurophysiol 94:2785-2796.

Bal T, McCormick DA (1996) What stops synchronized thalamocortical oscillations? Neuron 17:297-308.

Bal T, von Krosigk M, McCormick DA (1995a) Synaptic and membrane mechanisms underlying synchronized oscillations in the ferret lateral geniculate nucleus in vitro. J Physiol (Lond) 483:641-663.

Bal T, von Krosigk M, McCormick DA (1995b) Role of the ferret perigeniculate nucleus in the generation of synchronized oscillations in vitro. J Physiol 483:665-685.

Bartho P, Hirase H, Monconduit L, Zugaro M, Harris KD, Buzsaki G (2004) Characterization of neocortical principal cells and interneurons by network interactions and extracellular features. J Neurophysiol 92:600-608.

Berridge CW, Abercrombie ED (1999) Relationship between locus coeruleus discharge rates and rates of norepinephrine release within neocortex as assessed by in vivo microdialysis. Neuroscience 93:1263-1270.

Berridge CW, Foote SL (1991) Effects of locus ceruleus activation on electroencephalographic activity in neocortex and hippocampus. J Neurosci 11:3135-3145.

Berridge CW, Waterhouse BD (2003) The locus coeruleus-noradrenergic system: modulation of behavioral state and state-dependent cognitive processes. Brain Res Brain Res Rev 42:33-84.

Castro-Alamancos MA, Calcagnotto ME (2001) High-pass filtering of corticothalamic activity by neuromodulators released in the thalamus during arousal: in vitro and in vivo. J Neurophysiol 85:1489-1497.

Chapin JK, Nicolelis MA (1999) Principal component analysis of neuronal ensemble activity reveals multidimensional somatosensory representations. J Neurosci Methods 94:121-140.

Chiaia NL, Rhoades RW, Bennett-Clarke CA, Fish SE, Killackey HP (1991) Thalamic processing of vibrissal information in the rat. I. Afferent input to the medial ventral posterior and posterior nuclei. J Comp Neurol 314:201-216.

Churchland PM (1989) A neurocomputational perspective. Cambridge, MA: MIT.

Clayton EC, Rajkowski J, Cohen JD, Aston-Jones G (2004) Phasic activation of monkey locus ceruleus neurons by simple decisions in a forced-choice task. J Neurosci 24:9914-9920.

Csicsvari J, Hirase H, Czurko A, Buzsaki G (1998) Reliability and state dependence of pyramidal cell-interneuron synapses in the hippocampus: an ensemble approach in the behaving rat. Neuron 21:179-189.

Devilbiss DM, Waterhouse BD (2000) Norepinephrine exhibits two distinct profiles of action on sensory cortical neuron responses to excitatory synaptic stimuli. Synapse 37:273-282.

Devilbiss DM, Waterhouse BD (2002) Determination and quantification of pharmacological, physiological, or behavioral manipulations on ensembles of simultaneously recorded neurons in functionally related neural circuits. J Neurosci Methods 121:181-198.

Devilbiss DM, Waterhouse BD (2004) The effects of tonic locus ceruleus output on sensory-evoked responses of ventral posterior medial thalamic and barrel field cortical neurons in the awake rat. J Neurosci 24:10773-10785.

Diamond ME, Armstrong-James M, Ebner FF (1992) Somatic sensory responses in the rostral sector of the posterior group (POm) and in the 
ventral posterior medial nucleus (VPM) of the rat thalamus. J Comp Neurol 318:462-476.

Eggermont JJ (1992) Neural interaction in cat primary auditory cortex. Dependence on recording depth, electrode separation, and age. J Neurophysiol 68:1216-1228.

Eggermont JJ (1994) Neural interaction in cat primary auditory cortex II. Effects of sound stimulation. J Neurophysiol 71:246-270.

Erickson RP (1968) Stimulus coding in topographic and nontopographic afferent modalities: on the significance of the activity of individual sensory neurons. Psychol Rev 75:447-465.

Florin-Lechner SM, Druhan JP, Aston-Jones G, Valentino RJ (1996) Enhanced norepinephrine release in prefrontal cortex with burst stimulation of the locus coeruleus. Brain Res 742:89-97.

Foote SL, Freedman R, Oliver AP (1975) Effects of putative neurotransmitters on neuronal activity in monkey auditory cortex. Brain Res $86: 229-242$.

Foote SL, Aston-Jones G, Bloom FE (1980) Impulse activity of locus coeruleus neurons in awake rats and monkeys is a function of sensory stimulation and arousal. Proc Natl Acad Sci USA 77:3033-3037.

Foote SL, Bloom FE, Aston-Jones G (1983) Nucleus locus ceruleus: new evidence of anatomical and physiological specificity. Physiol Rev 63:844-914.

Friedberg MH, Lee SM, Ebner FF (2004) The contribution of the principal and spinal trigeminal nuclei to the receptive field properties of thalamic VPM neurons in the rat. J Neurocytol 33:75-85.

George MJ (1992) Modification of receptive fields of posteriomedial barrel subfield neocortical single units by known concentrations of iontophoresed noradrenaline in the rat. Int J Neurosci 65:69-81.

Gerstein GL, Bedenbaugh P, Aertsen MH (1989) Neuronal assemblies. IEEE Trans Biomed Eng 36:4-14.

Grant SJ, Highfield DA (1991) Extracellular characteristics of putative cholinergic neurons in the rat laterodorsal tegmental nucleus. Brain Res 559:64-74

Hallanger AE, Levey AI, Lee HJ, Rye DB, Wainer BH (1987) The origins of cholinergic and other subcortical afferents to the thalamus in the rat. J Comp Neurol 262:105-124.

Harris KD (2005) Neural signatures of cell assembly organization. Nat Rev Neurosci 6:399-407.

Holdefer RN, Jacobs BL (1994) Phasic stimulation of the locus coeruleus: effects on activity in the lateral geniculate nucleus. Exp Brain Res 100:444-452.

Hurley LM, Devilbiss DM, Waterhouse BD (2004) A matter of focus: monoaminergic modulation of stimulus coding in mammalian sensory networks. Curr Opin Neurobiol 14:488-495.

Ito M (1981) Some quantitative aspects of vibrissa-driven neuronal responses in rat neocortex. J Neurophysiol 46:705-715.

Ito M (1988) Response properties and topography of vibrissa-sensitive VPM neurons in the rat. J Neurophysiol 60:1181-1197.

Ivanova S, Rajkowski J, Silakov V, Watanabe T, Aston-Jones G (1997) Local chemomanipulations of locus coeruleus (LC) activity in monkeys alter cortical event-related potentials (ERPs) and task performance. Soc Neurosci Abstr 23:1587.

Jones BE (1990) Immunohistochemical study of choline acetyltransferaseimmunoreactive processes and cells innervating the pontomedullary reticular formation in the rat. J Comp Neurol 295:485-514.

Jones BE, Yang TZ (1985) The efferent projections from the reticular formation and the locus coeruleus studied by anterograde and retrograde axonal transport in the rat. J Comp Neurol 242:56-92.

Katchalsky AK, Rowland V, Blumenthal R (1974) Dynamic patterns of brain cell assemblies: a report based on an NRP work session held May 14-16, 1972, and updated by participants. Cambridge, MA: MIT.

Kohlmeier KA, Reiner PB (1999) Noradrenaline excites non-cholinergic laterodorsal tegmental neurons via two distinct mechanisms. Neuroscience 93:619-630.

Kossl M, Vater M (1989) Noradrenaline enhances temporal auditory contrast and neuronal timing precision in the cochlear nucleus of the mustached bat. J Neurosci 9:4169-4178.

Lampl I, Reichova I, Ferster D (1999) Synchronous membrane potential fluctuations in neurons of the cat visual cortex. Neuron 22:361-374.

Laurent G, Wehr M, Davidowitz H (1996) Temporal representations of odors in an olfactory network. J Neurosci 16:3837-3847.

Lee KH, McCormick DA (1996) Abolition of spindle oscillations by seroto- nin and norepinephrine in the ferret lateral geniculate and perigeniculate nuclei in vitro. Neuron 17:309-321.

Manunta Y, Edeline JM (1997) Effects of noradrenaline on frequency tuning of rat auditory cortex neurons. Eur J Neurosci 9:833-847.

Marshall L, Henze DA, Hirase H, Leinekugel X, Dragoi G, Buzsaki G (2002) Hippocampal pyramidal cell-interneuron spike transmission is frequency dependent and responsible for place modulation of interneuron discharge. J Neurosci 22:RC197.

McCormick DA (1992) Cellular mechanisms underlying cholinergic and noradrenergic modulation of neuronal firing mode in the cat and guinea pig dorsal lateral geniculate nucleus. J Neurosci 12:278-289.

McCormick DA, Pape HC (1988) Acetylcholine inhibits identified interneurons in the cat lateral geniculate nucleus. Nature 334:246-248.

McCormick DA, Pape HC (1990) Noradrenergic and serotonergic modulation of a hyperpolarization-activated cation current in thalamic relay neurones. J Physiol (Lond) 431:319-342.

McCormick DA, Prince DA (1988) Noradrenergic modulation of firing pattern in guinea pig and cat thalamic neurons, in vitro. J Neurophysiol 59:978-996.

McCormick DA, von Krosigk M (1992) Corticothalamic activation modulates thalamic firing through glutamate "metabotropic" receptors. Proc Natl Acad Sci USA 89:2774-2778.

McCormick DA, Wang Z (1991) Serotonin and noradrenaline excite GABAergic neurones of the guinea-pig and cat nucleus reticularis thalami. J Physiol (Lond) 442:235-255.

McCormick DA, Pape HC, Williamson A (1991) Actions of norepinephrine in the cerebral cortex and thalamus: implications for function of the central noradrenergic system. Prog Brain Res 88:293-305.

Nicolelis MA (1996) Beyond maps: a dynamic view of the somatosensory system. Braz J Med Biol Res 29:401-412.

Nicolelis MA, Chapin JK (1994) Spatiotemporal structure of somatosensory responses of many-neuron ensembles in the rat ventral posterior medial nucleus of the thalamus. J Neurosci 14:3511-3532.

Nicolelis MA, Baccala LA, Lin RC, Chapin JK (1995) Sensorimotor encoding by synchronous neural ensemble activity at multiple levels of the somatosensory system. Science 268:1353-1358.

Nieuwenhuis S, Aston-Jones G, Cohen JD (2005) Decision making, the P3, and the locus coeruleus-norepinephrine system. Psychol Bull 131: $510-532$.

Page ME, Abercrombie ED (1997) An analysis of the effects of acute and chronic fluoxetine on extracellular norepinephrine in the rat hippocampus during stress. Neuropsychopharmacology 16:419-425.

Panzeri S, Petersen RS, Schultz SR, Lebedev M, Diamond ME (2001) The role of spike timing in the coding of stimulus location in rat somatosensory cortex. Neuron 29:769-777.

Panzeri S, Petroni F, Petersen RS, Diamond ME (2003) Decoding neuronal population activity in rat somatosensory cortex: role of columnar organization. Cereb Cortex 13:45-52.

Petersen RS, Diamond ME (2000) Spatial-temporal distribution of whisker-evoked activity in rat somatosensory cortex and the coding of stimulus location. J Neurosci 20:6135-6143.

Petersen RS, Panzeri S, Diamond ME (2001) Population coding of stimulus location in rat somatosensory cortex. Neuron 32:503-514.

Petersen RS, Panzeri S, Diamond ME (2002) Population coding in somatosensory cortex. Curr Opin Neurobiol 12:441-447.

Rajkowski J, Kubiak P, Aston-Jones G (1994) Locus coeruleus activity in monkey: phasic and tonic changes are associated with altered vigilance. Brain Res Bull 35:607-616.

Ritz R, Sejnowski TJ (1997) Synchronous oscillatory activity in sensory systems: new vistas on mechanisms. Curr Opin Neurobiol 7:536-546.

Rogawski MA, Aghajanian GK (1980) Norepinephrine and serotonin: opposite effects on the activity of lateral geniculate neurons evoked by optic pathway stimulation. Exp Neurol 69:678-694.

Rudolph M, Destexhe A (2003) Tuning neocortical pyramidal neurons between integrators and coincidence detectors. J Comput Neurosci 14:239-251.

Salinas E, Sejnowski TJ (2000) Impact of correlated synaptic input on output firing rate and variability in simple neuronal models. J Neurosci 20:6193-6209.

Sato H, Kayama Y (1983) Effects of noradrenaline applied iontophoretically on rat superior collicular neurons. Brain Res Bull 10:453-457.

Satoh K, Fibiger HC (1986) Cholinergic neurons of the laterodorsal teg- 
mental nucleus: efferent and afferent connections. J Comp Neurol 253:277-302.

Sejnowski TJ, Paulsen O (2006) Network oscillations: emerging computational principles. J Neurosci 26:1673-1676.

Shipley MT (1974) Response characteristics of single units in the rat's trigeminal nuclei to vibrissa displacements. J Neurophysiol 37:73-90.

Shoykhet M, Doherty D, Simons DJ (2000) Coding of deflection velocity and amplitude by whisker primary afferent neurons: implications for higher level processing. Somatosens Mot Res 17:171-180.

Sillito AM, Jones HE, Gerstein GL, West DC (1994) Feature-linked synchronization of thalamic relay cell firing induced by feedback from the visual cortex. Nature 369:479-482.

Simons DJ (1978) Response properties of vibrissa units in rat SI somatosensory neocortex. J Neurophysiol 41:798-820.

Simpson KL, Altman DW, Wang L, Kirifides ML, Lin RCS, Waterhouse BD (1997) Lateralization and functional organization of the locus coeruleus projection to the trigeminal somatosensory pathway in rat. J Comp Neurol 385:135-148.

Singer W (1999) Neuronal synchrony: a versatile code for the definition of relations? Neuron 24:49-65.

Snow PJ, Andre P, Pompeiano O (1999) Effects of locus coeruleus stimulation on the responses of SI neurons of the rat to controlled natural and electrical stimulation of the skin. Arch Ital Biol 137:1-28.

Timofeeva E, Lavallee P, Arsenault D, Deschenes M (2004) Synthesis of multiwhisker-receptive fields in subcortical stations of the vibrissa system. J Neurophysiol 91:1510-1515.

Usher M, Cohen JD, Servan-Schreiber D, Rajkowski J, Aston-Jones G (1999) The role of locus coeruleus in the regulation of cognitive performance. Science 283:549-554.

Van der Loos H (1976) Barreloids in mouse somatosensory thalamus. Neurosci Lett 2:1-6.

Waite PM (1973) The responses of cells in the rat thalamus to mechanical movements of the whiskers. J Physiol (Lond) 228:541-561.

Wang BR, Ohara PT (1993) Convergent projections of trigeminal afferents from the principal nucleus and subnucleus interpolaris upon rat ventral posteromedial thalamic neurons. Brain Res 629:253-259.

Waterhouse BD, Woodward DJ (1980) Interaction of norepinephrine with cerebrocortical activity evoked by stimulation of somatosensory afferent pathways in the rat. Exp Neurol 67:11-34.

Waterhouse BD, Azizi SA, Burne RA, Woodward DJ (1990) Modulation of rat cortical area 17 neuronal responses to moving visual stimuli during norepinephrine and serotonin microiontophoresis. Brain Res 514:276-292.

Wilent WB, Contreras D (2004) Synaptic responses to whisker deflections in rat barrel cortex as a function of cortical layer and stimulus intensity. J Neurosci 24:3985-3998. 